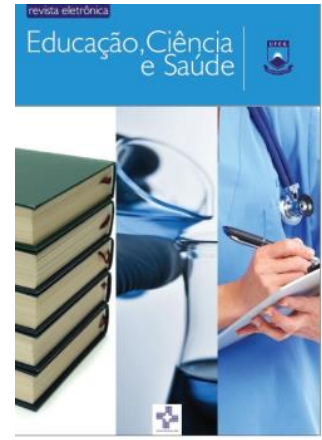

EDUCAÇÃO CIÊNCIA E SAÚDE

http://dx.doi.org/10.20438/ecs.v7i1.256

\title{
AVALIAÇÃO DE EXTRATOS DE Sida planicaulis Cav. POR MEIO DO BIOENSAIO COM Artemia salina Leach
}

\author{
Gabriel Ferreira Marques ${ }^{1}$, Thiago Araújo de Medeiros Brito², Ana Laura de \\ Cabral Sobreira ${ }^{3}$, Danielly Albuquerque da Costa ${ }^{4}$, Fernando de Sousa \\ Oliveira ${ }^{5}$ \\ ${ }^{1}$ Mestrando em Ciências Naturais e Biotecnologia, Centro de Educação e Saúde, Universidade \\ Federal de Campina Grande, Cuité, PB, Brasil. \\ ${ }^{2}$ Mestrando em Produtos Naturais e Sintéticos Bioativos, Centro de Ciências da Saúde, \\ Universidade Federal da Paraíba, João Pessoa, PB, Brasil. \\ ${ }^{3}$ Doutoranda em Desenvolvimento e Inovação Tecnológica em Medicamentos, Centro de \\ Ciências da Saúde, Universidade Federal da Paraíba, João Pessoa, PB, Brasil. \\ ${ }^{4}$ Prof ${ }^{a}$. do Departamento de Fisiologia e Patologia, Centro de Ciências da Saúde, Universidade \\ Federal da Paraíba, João Pessoa, PB, Brasil. \\ ${ }^{5}$ Prof. do Centro de Educação e Saúde, Universidade Federal de Campina Grande, Cuité, PB, \\ Brasil. \\ Email para correspondência: fernandoufcg@hotmail.com
}

\begin{abstract}
Resumo
As plantas medicinais têm sido utilizadas mundialmente no tratamento de várias enfermidades, porém muitas dessas espécies não foram suficientemente estudadas. Essas plantas podem conter substâncias tóxicas capazes de causar danos à saúde da população. Objetivou-se avaliar a atividade do extrato etanólico bruto (EEB) e das fases clorofórmica e hidroalcoólica oriundas das partes aéreas de Sida planicaulis Cav., por determinação da concentração letal $50 \%$ (CL50) através do ensaio com Artemia salina Leach, visando sua utilização em estudos farmacológicos posteriores. Cada concentração foi testada em três etapas, e cada uma delas durou 24 horas, a fim de que o crescimento das artemias fosse padronizado nesse intervalo de tempo. Para análise estatística, utilizou-se o Software POLO (Probit Or LOgit). Após a realização dos testes foi possível elaborar uma tabela relacionando a viabilidade das artemias às concentrações em $\mu \mathrm{g} / \mathrm{mL}$ dos extratos. Sendo assim, obtendo-se a $\mathrm{CL}_{50}$ para o EEB de 235,057 $\mu \mathrm{g} / \mathrm{mL}(105,635-329,472 \mu \mathrm{g} / \mathrm{mL})$, para a fase clorofórmica de $234,221 \mu \mathrm{g} / \mathrm{mL}$ $(190,943-268,182 \mu \mathrm{g} / \mathrm{mL})$, e de $704,195 \mu \mathrm{g} / \mathrm{mL}$ para a fase hidroalcoólica. Contudo, a análise demonstrou que o EEB e a fase clorofórmica demonstraram toxicidade moderada, enquanto que, a hidroalcoólica apresentou leve toxicidade em artemias.
\end{abstract}

Palavras-chave: Anostraca, Plantas Medicinais, Malvaceae.

\section{Abstract}

Educ. Ci. e Saúde, v. 7, n. 1, p. 132-144, jan./jun., 2020 


\begin{abstract}
Medicinal plants have been used worldwide in the treatment of various diseases, but many of these species have not been sufficiently studied. These plants may contain toxic substances capable of causing damage to the health of the population. The objective was to evaluate the activity of the crude ethanolic extract (BSE) and the chloroformic and hydroalcoholic phases from the aerial parts of Sida planicaulis Cav., by determining the $50 \%$ lethal concentration $\left(\mathrm{LC}_{50}\right)$ through the trial with Artemia salina Leach, aiming at its use in later pharmacological studies. Each concentration was tested in three stages, and each one lasted 24 hours, so that the growth of the brine shrimp was standardized in that time interval. For statistical analysis, the POLO software (Probit Or LOgit) was used. After performing the tests, it was possible to create a table relating the viability of the brine shrimp to the $\mu \mathrm{g} / \mathrm{mL}$ concentrations of the extracts. Thus, obtaining the $\mathrm{LC}_{50}$ for the BSE of $235.057 \mu \mathrm{g} / \mathrm{mL}(105.635-329.472 \mu \mathrm{g} / \mathrm{mL})$, for the chloroform phase of $234.221 \mu \mathrm{g} / \mathrm{mL}(190.943-268.182 \mu \mathrm{g} / \mathrm{mL})$, and of $704.195 \mu \mathrm{g} / \mathrm{mL}$ for the hydroalcoholic phase. However, the analysis showed that BSE and the chloroform phase showed moderate toxicity, while the hydroalcoholic showed mild toxicity in brine shrimp.
\end{abstract}

Keywords: Anostraca, Medicinal Plants, Malvaceae.

\title{
1 Introdução
}

De acordo com a Organização Mundial de Saúde, cerca de $60-80 \%$ da população mundial, nos países em desenvolvimento, dependem essencialmente de plantas para cuidar de sua saúde. Isso ocorre, principalmente, devido à pobreza e falta de acesso a medicamentos industrializados. Entretanto, mesmo com a diversidade genética vegetal no mundo sendo bastante expressiva, poucas são as espécies (15-17\%) que têm sido cientificamente estudadas para a avaliação de suas qualidades, segurança e eficácia (SILVA et al., 2015).

Como todo medicamento, as plantas medicinais também necessitam de estudos científicos e procedimentos laboratoriais para quantificar e qualificar suas características de eficácia e faixa de toxicidade nos seres vivos, pois mesmo sendo um medicamento a base de substâncias naturais, não deixa de apresentar efeitos adversos quando encontrado em concentrações maiores ou preparo com métodos empíricos (OLIVEIRA et al., 2020).

Os testes de toxicidade são elaborados com objetivo de avaliar ou prever os efeitos de substâncias tóxicas nos sistemas biológicos, assim como, averiguarem a toxicidade relativa das substâncias em determinadas doses ou concentrações (SILVA et al., 2016).

O uso de ensaios biológicos para avaliação da bioatividade de extratos, frações e compostos isolados de plantas tem sido, frequentemente, incorporado à identificação e monitoramento de substâncias vegetais 
potencialmente tóxicas. Dentre esses bioensaios, encontra-se a toxicidade sobre Artemia salina Leach, um microcrustáceo de água salgada muito utilizado como bioindicador em ensaios de toxicidade aguda de extratos de plantas, por apresentar um baixo custo financeiro e rápida execução. A literatura descreve a utilização das artemias para detectar compostos bioativos em extratos vegetais, como também expressar a sua toxicidade (DOS SANTOS et al., 2018; ARRUDA et al., 2019).

Dentre as plantas medicinais que apresentam estudos científicos comprovando os seus benefícios para saúde humana, pode-se citar as pertencentes à família Malvaceae. Entre os gêneros desta família, ressalta-se Sida, que em estudos anteriores, demonstrou vários efeitos farmacológicos, como: anti-inflamatório, hepatoprotetor, antimicrobiano, vaso-relaxante e analgésico (CHAVES et al., 2013; MOMIN et al., 2014; B; MUTHURAMAN, 2014; GALAL et al., 2015; CHAVES et al., 2017).

A espécie Sida planicaulis Cav. é conhecida como vassoura ou guanxuma e está distribuída por todo o território brasileiro, principalmente na região Nordeste. Tradicionalmente, destaca-se por tratar dor no corpo, sendo ainda pouco estudada no tocante aos aspectos químicos e farmacológicos (BRITO; SENNA-VALLE, 2012).

Dentre os raros estudos realizados com Sida planicaulis Cav., observa-se uma pesquisa fitoquímica realizada com o extrato etanólico bruto (EEB) e as fases hexânica, clorofórmica, acetato de etila e hidroalcoólica obtidos das partes aéreas desta espécie, que detectou uma diversidade de metabólitos secundários, tais como: alcaloides, esteroides, flavonoides, taninos e triterpeno (SOBREIRA et al., 2018). Além destes, uma investigação do potencial antifúngico de Sida planicaulis Cav. frente a fungos filamentosos, apresentou uma atividade moderada dessa espécie contra o fungo Trichophyton mentagrophytes, fato associado à presença desses metabólitos secundários na planta (SOBREIRA et al., 2019).

Nesse sentido, a investigação da toxicidade de Sida planicaulis Cav. torna-se fundamental para maior segurança quanto a sua utilização, especialmente quanto ao seu uso tradicional. 
O objetivo deste trabalho foi avaliar a atividade do EEB e das fases clorofórmica e hidroalcoólica oriundas das partes aéreas de Sida planicaulis Cav., por determinação da concentração letal $50 \%\left(\mathrm{CL}_{50}\right)$ por meio do ensaio com Artemia salina Leach, visando sua utilização em estudos farmacológicos posteriores.

\section{Material e métodos}

\subsection{Tipo de estudo}

Corresponde a um estudo experimental, quantitativo, realizado por dois graduandos do curso de Farmácia da Universidade Federal de Campina Grande, orientados por um docente da mesma instituição.

A pesquisa teve a colaboração de uma docente e uma pós-graduanda da Universidade Federal da Paraíba que, além da co-orientação prestada, produziram e forneceram as extrações vegetais testadas. As pesquisas foram procedidas de janeiro a dezembro de 2017.

\subsection{Local da pesquisa}

As atividades de pesquisa foram desenvolvidas no Laboratório de Farmacologia e Toxicologia do Centro de Educação e Saúde, da Universidade Federal de Campina Grande - Campus Cuité, Paraíba.

\subsection{Procedimento da pesquisa}

\subsubsection{Incubadora}

A incubadora para eclosão dos cistos de Artemia salina Leach consistiu de um recipiente retangular de vidro com uma divisória com orifícios de aproximadamente $0,02 \mathrm{~cm}$ de espessura e distribuídos uniformemente. $O$ meio para eclosão do cisto foi uma solução salina feita com $39 \mathrm{~g}$ de sal marinho e $1 \mathrm{~L}$ de água destilada, em um pH entre 8,0 a 8,5 (Figura 1). 


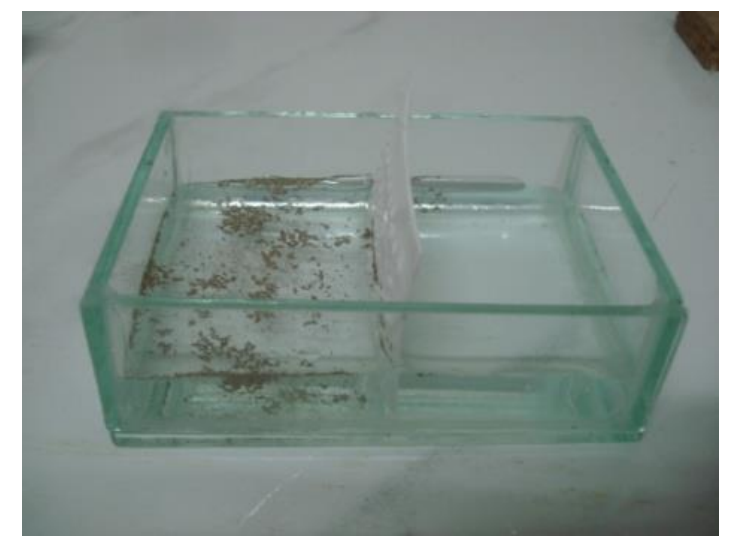

Figura 1: Incubadora com divisória contendo orifícios.

Fonte: Próprio autor

\subsubsection{Obtenção do extrato etanólico bruto e fases de Sida planicaulis}

O extrato bruto foi obtido das partes aéreas de Sida planicaulis Cav. por maceração em etanol a $96 \%$ e, posteriormente, submetido a extração líquidolíquido utilizando solventes com gradiente crescente de polaridade (hexano, clorofórmio, acetato de etila). Para o estudo da toxicidade utilizou-se o EEB, fase clorofórmica e a fase hidroalcoólica, que foram armazenadas em recipiente fechado sob temperatura de $15-20^{\circ} \mathrm{C}$.

\subsubsection{Preparação da solução-mãe}

Os extratos e fases testados foram solubilizados em solução salina artificial a fim de se obter uma solução-mãe de $2 \mathrm{mg} / \mathrm{mL}$. O volume da solução-mãe foi determinado pela quantidade de tubos e a concentração que se desejou alcançar até um volume final de $5 \mathrm{~mL}$ por tudo de ensaio. As amostras foram obtidas do EEB. A primeira amostra foi EEB, a segunda consistiu da fase clorofórmica e a terceira foi preparada a partir da fase hidroalcoólica. As fases foram solubilizadas em $5 \mathrm{~mL}$ de solução salina. Foi utilizado $30 \mu \mathrm{L}$ de solvente (dimetilsulfóxido) para auxiliar na solubilização dos extratos, visto que apresentavam caráter lipofílico.

Após determinar a concentração mínima de $250 \mu \mathrm{L}$ do EEB e fases clorofórmica e hidroalcoólica, iniciaram-se os testes de toxicidade aguda, no qual foram colocados em tubos de ensaio concentrações de 1900, 3750 e 7500 $\mu \mathrm{L}$ de solução-mãe para o extrato e cada fase. Em seguida, foi adicionado a 
cada tubo $5 \mathrm{~mL}$ da solução salina, obtendo-se assim concentrações de 250, 500 e $1000 \mu \mathrm{g} / \mathrm{mL}$, respectivamente.

\subsubsection{Ensaios}

Para a realização dos ensaios foi adotada a metodologia descrita por Meyer et al. (1982). Cada concentração foi testada em três etapas, em que cada uma delas durou 24 horas, a fim de que o crescimento das artemias fosse padronizado nesse intervalo de tempo.

A primeira etapa baseava-se na incubação das artemias. Foram colocados os cistos na incubadora dividida por uma tela semipermeável, com uma das metades recoberta por um papel alumínio (Figura 2).

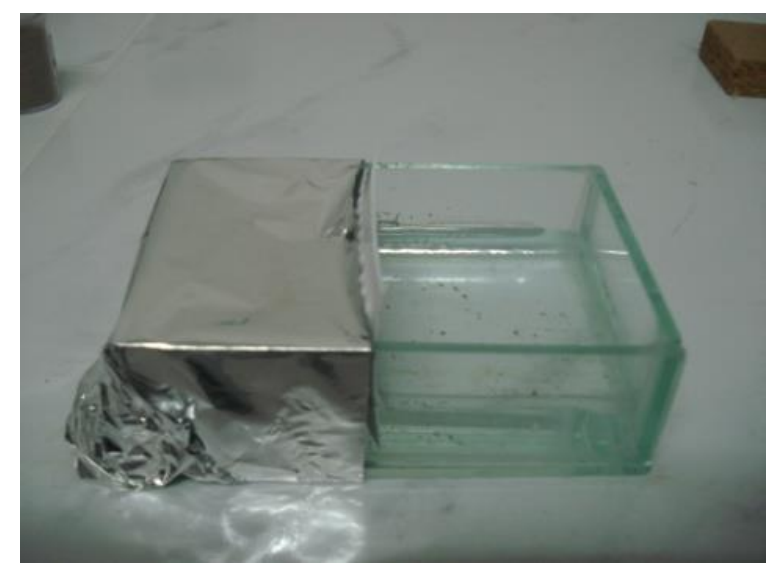

Figura 2: Parte recoberta do sistema contendo os cistos.

Fonte: Próprio autor

A incubadora foi mantida sob iluminação de uma lâmpada incandescente (40 W), a fim de que as artemias, quando eclodidas, fossem atraídas pela luz na região descoberta para que pudessem ser coletadas (Figura 3). 


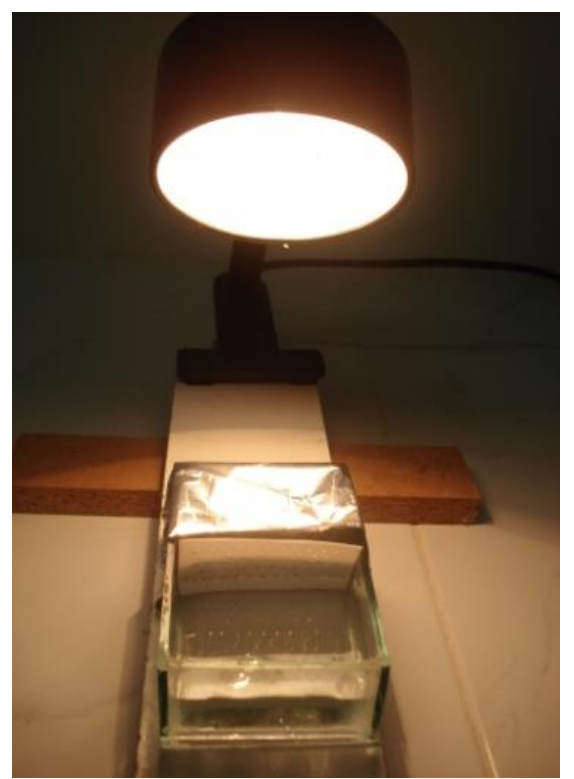

Figura 3: Incubadora sob exposição à luz.

Fonte: Próprio autor

A segunda etapa consistiu na exposição das artemias em diferentes volumes da solução-mãe (1900, 3750 e $7500 \mu \mathrm{L})$. Em cada tubo de ensaio foram adicionadas 10 artemias e em seguida $5 \mathrm{~mL}$ de solução salina, criandose assim concentrações de 250, 500 e $1000 \mu \mathrm{g} / \mathrm{mL}$. Cada concentração foi testada em triplicata e em três testes posteriores, resultando em nove tubos de ensaio para cada concentração. Na terceira etapa foi procedida a contagem de artemias vivas e mortas para posterior análise em programas estatísticos e determinação da CL50.

\subsubsection{Grupo controle}

Os grupos controles do EEB e das fases clorofórmica e hidroalcoólica foram preparados contendo a solução salina com as artemias e $30 \mu \mathrm{L}$ de solvente (dimetilsulfóxido), já que este foi utilizado para a solubilização do extrato e das fases. A quantidade de solvente foi estabelecida a partir da quantidade suficiente para solubilizar as fases sem causar toxicidade às artemias.

\subsubsection{Análise estatística}

Os valores de $\mathrm{CL}_{50}$ foram calculados por meio da expressão dos resultados como uma percentagem dos controles, por regressão não-linear com intervalo de confiança de 95\%, para análise estatística, utilizou-se o software POLO (Probit Or LOgit). 


\section{$3 \quad$ Resultados e discussão}

A análise dos resultados obtidos no biosensaio com $A$. salina mostrou-se eficiente, uma vez que, pode-se avaliar a toxicidade do EEB e das fases clorofórmica e hidroalcoólica de S. planicaulis Cav.

Para o EEB observou-se que, com o aumento das concentrações testadas, houve aumento da letalidade das artemias, como demonstrado na tabela 1.

Tabela 1: Porcentagem de letalidade nas artemias em relação ao aumento da concentração EEB de Sida planicaulis Cav.

\begin{tabular}{|c|c|c|c|c|}
\hline Concentração & Controle & $250 \mu \mathrm{g} / \mathrm{mL}$ & $500 \mu \mathrm{g} / \mathrm{mL}$ & $1000 \mu \mathrm{g} / \mathrm{mL}$ \\
\hline \multirow{2}{*}{$\begin{array}{c}\text { Letalidade nas } \\
\text { artemias }\end{array}$} & $0 \%$ & $40 \%$ & $60 \%$ & $80 \%$ \\
\cline { 2 - 5 } & $0 \%$ & $40 \%$ & $60 \%$ & $80 \%$ \\
\cline { 2 - 5 } & $0 \%$ & $40 \%$ & $60 \%$ & $90 \%$ \\
\hline
\end{tabular}

Fonte: Elaboração própria

Esses dados foram plotados obtendo-se o gráfico 1. Além desse, foi possível calcular uma $\mathrm{CL}_{50}$ de $234,22 \mu \mathrm{g} / \mathrm{mL}$ com intervalo de confiança de $190,94-268,18 \mu \mathrm{g} / \mathrm{mL}$.

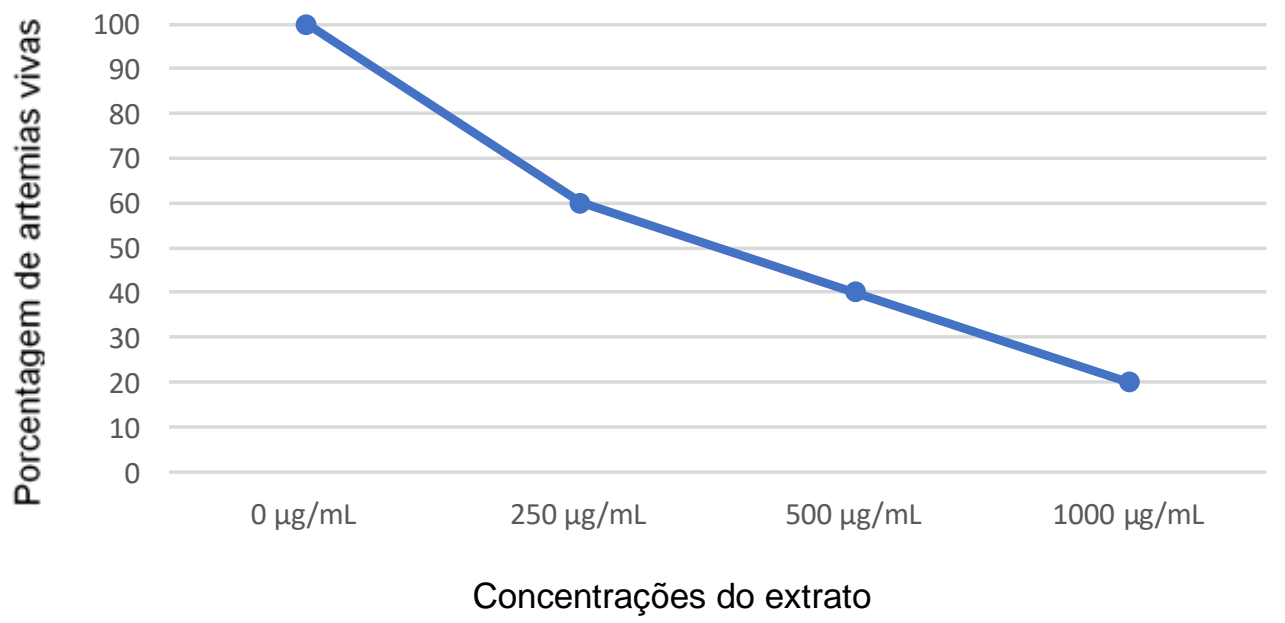

Gráfico 1: Viabilidade das artemias em relação à concentração do EEB.

Fonte: Elaboração própria 
Para a fase clorofórmica, os resultados estão demostrados na tabela 2, em que o aumento da concentração foi acompanhado de aumento da letalidade das artemias.

Tabela 2: Porcentagem de letalidade nas artemias em relação ao aumento da concentração da fase clorofórmica do EEB de Sida planicaulis Cav.

\begin{tabular}{|c|c|c|c|c|}
\hline Concentração & Controle & $250 \mu \mathrm{g} / \mathrm{mL}$ & $500 \mu \mathrm{g} / \mathrm{mL}$ & $1000 \mu \mathrm{g} / \mathrm{mL}$ \\
\hline \multirow{3}{*}{$\begin{array}{l}\text { Letalidade nas } \\
\text { artemias }\end{array}$} & $0 \%$ & $50 \%$ & $80 \%$ & $100 \%$ \\
\hline & $0 \%$ & $50 \%$ & $80 \%$ & $100 \%$ \\
\hline & $0 \%$ & $50 \%$ & $80 \%$ & $100 \%$ \\
\hline
\end{tabular}

Fonte: Elaboração própria

Com dados da fase clorofórmica, obteve-se o gráfico 2 e uma $\mathrm{CL}_{50}$ de $235,06 \mu \mathrm{g} / \mathrm{mL}(105,64-329,47 \mu \mathrm{g} / \mathrm{mL})$, resultados semelhantes aos encontrados com o EEB.

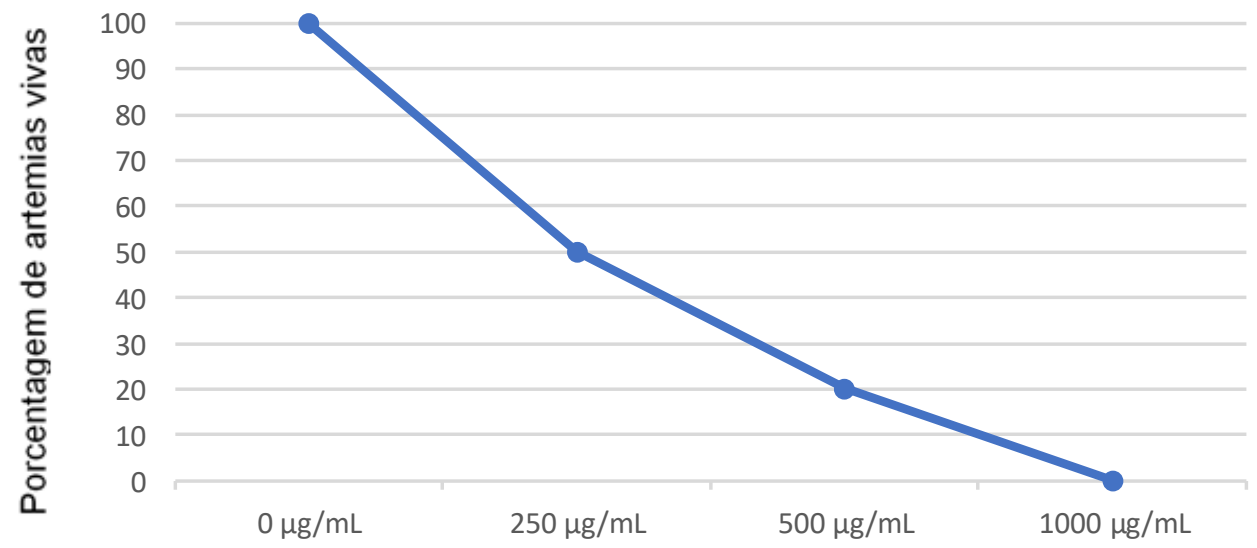

Concentrações da fase

Gráfico 2: Viabilidade das artemias em relação à concentração da fase clorofórmica.

Fonte: Elaboração própria

A fase hidroalcoólica do EEB causou, da mesma forma, aumento de letalidade das artemias a partir de aumento das concentrações. Esses resultados estão descritos na tabela 3 . 
Tabela 3: Porcentagem de letalidade nas artemias em relação ao aumento da concentração da fase hidroalcoólica do EEB de Sida planicaulis Cav.

\begin{tabular}{|c|c|c|c|c|}
\hline Concentração & Controle & $250 \mu \mathrm{g} / \mathrm{mL}$ & $500 \mu \mathrm{g} / \mathrm{mL}$ & $1000 \mu \mathrm{g} / \mathrm{mL}$ \\
\hline \multirow{2}{*}{$\begin{array}{c}\text { Letalidade nas } \\
\text { artemias }\end{array}$} & $0 \%$ & $33 \%$ & $60 \%$ & $100 \%$ \\
\cline { 2 - 5 } & $0 \%$ & $0 \%$ & $27 \%$ & $47 \%$ \\
\cline { 2 - 5 } & $0 \%$ & $13 \%$ & $27 \%$ & $40 \%$ \\
\hline
\end{tabular}

Fonte: Elaboração própria

Diferentemente do EEB e da fase clorofórmica, a fase hidroalcoólica demosntrou uma $\mathrm{CL}_{50}$ de $704,20 \mu \mathrm{g} / \mathrm{mL}(593,32-884,89 \mu \mathrm{g} / \mathrm{mL})$, dessa forma, apresentrando uma gráfico diferente dos outros extratos testados, bem como, uma toxicidade significativamente inferior.

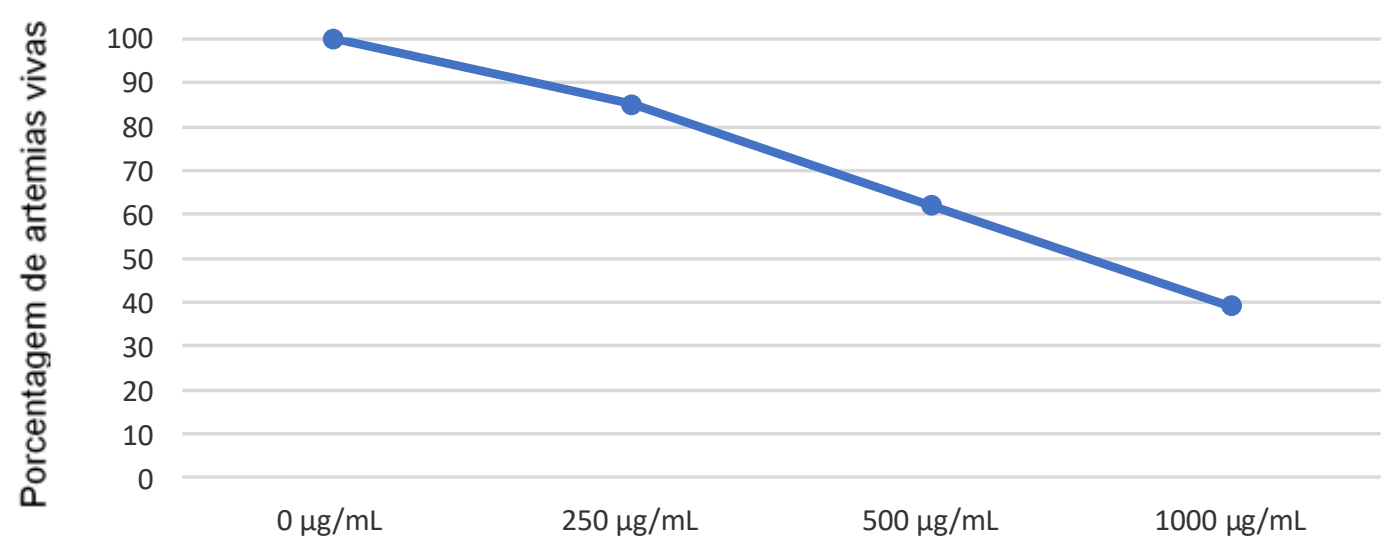

Concentrações do extrato

Gráfico 3: Viabilidade das artemias em relação à concentração da fase hidroalcoólica.

Fonte: Elaboração própria

Meyer et al. (1982) estabeleceram uma relação entre o grau de toxicidade e a dose letal média $\left(C_{50}\right)$, apresentada por extratos de plantas sobre a $A$. salina, desde então, considera-se que quando são verificados valores abaixo de $1000 \mu \mathrm{g} / \mathrm{mL}$ estes são considerados com relativa toxicidade.

Em uma triagem fitoquímica realizada por Sobreira et al. (2018), o EEB apresentou como constituintes majoritários, principalmente, alcaloides e esteroides. Possivelmente, esses constituintes estejam envolvidos com a $\mathrm{CL}_{50}$ do EEB.

A fase clorofórmica do EEB de S. planicaulis Cav., por sua vez, destacouse pela presença de constituintes semi-polares, como por exemplo: os 
alcaloides e esteroides, de forma semelhante ao EEB (SOBREIRA et al., 2018). Esses constituintes, em especial os alcaloides, podem estar envolvidos com as $\mathrm{CL}_{50}$ encontradas, por serem metabólitos secundários conhecidos na literatura por sua elevada toxicidade (SIMÕES et al., 2010; HYACIENTH; DE ALMEIDA, 2015).

A fase hidroalcoólica apresentou uma concentração de substâncias polares, a exemplo de taninos e saponinas (SOBREIRA et al., 2018). Esses constituintes podem estar envolvidos com a $\mathrm{CL}_{50}$ encontrada neste estudo.

De acordo com Nguta et al. (2011) amostras com valores de $\mathrm{CL}_{50}$ inferiores $100 \mu \mathrm{g} / \mathrm{mL}$ são considerados altamente tóxicas, valores entre $100 \mathrm{e}$ $500 \mu \mathrm{g} / \mathrm{mL}$ são moderadamente tóxicas, entre 500 e $1000 \mu \mathrm{g} / \mathrm{mL}$ são suavemente tóxicas e acima de $1000 \mu \mathrm{g} / \mathrm{mL}$ são atóxicas. Sendo assim, o EEB e a fase clorofórmica apresentaram toxicidade moderada, enquanto que, com a fase hidroalcoólica foi observada uma leve toxicidade em artemias.

Esses dados encontrados de Sida planicaulis Cav. servem como motivação para estudos futuros envolvendo a aplicação dessa espécie em testes farmacológico. Entretanto, é importante enfatizar que a sazonalidade, desenvolvimento vegetal, temperatura, disponibilidade hídrica, incidência de radiação ultravioleta, disponibilidade de nutrientes, altitude e índice de poluição são fatores que influenciam na produção dos metabólitos secundários nas plantas, bem como, em seu efeito toxicológico (Souza et al., 2018).

\section{Conclusão}

A utilização de bioensaios para o monitoramento da bioatividade de extratos se apresentou como um bom indicador de toxicidade. Com valores de $\mathrm{CL}_{50}$, das três extrações testadas, o EEB e a fase clorofórmica apresentaram resultados semelhantes, enquanto que a fase hidroalcoólica demonstrou a maior $\mathrm{CL}_{50} \mathrm{e}$, consequentemente, a menor letalidade em artemias.

Contudo, evidencia-se a necessidade de estudos mais aprofundados por meio de testes farmacológicos e toxicológicos específicos, auxiliando na propagação de conhecimento acerca da espécie Sida planicaulis Cav., tendo como resultado final a prevenção e promoção da saúde. 


\section{Referências}

ARRUDA, Ana Carolina de Souza Toesca et al. Análise fitoquímica e toxicológica de Pothomorphe umbellata. Brazilian Journal of Natural Sciences, v. 1, n. 2, p. 1-9, fev. 2019.

B, Srinithya, MUTHURAMAN, Meenakshi Sundaram. An overview on the biological perspectives of Sida cordifolia Linn. International Journal of Pharmacy and Pharmaceutical Sciences, v. 6, n. 11, p. 15-17, out. 2014.

BRITO, Mariana Reis de; SENNA-VALLE, Luci de. Diversity of plant knowledge in a "Caiçara" community from the Brazilian Atlantic Forest coast. Acta Botanica Brasilica, v. 26 , n. 4 , p. 735-747, out./dez. 2012.

CHAVES, Otemberg Souza et al. Alkaloids and phenolic compounds from Sida rhombifolia L. (Malvaceae) and vasorelaxant activity of two Indoquinoline alkaloids. Molecules, v. 22, n. 1, p. 94-103, jan. 2017.

CHAVES, Otemberg Souza et al. Secondary metabolites from Sida rhombifolia L. (Malvaceae) and the vasorelaxant activity of cryptolepinone. Molecules, v.18, n. 3, p. 2769-2777, jan. 2013.

DOS SANTOS, Antônio Ruan Souto et al. Avaliação toxicológica frente as larvas da Artemia salina Leach do 1,2:3,4-di-O-isopropilideno-alfa- $D$-galactopiranose. Educação, Ciência e Saúde, v. 5, n. 5, p. 1-14, jan./jun. 2018.

GALAL, Ahmed et al. Sida cordifolia, a traditional herb in modern perspective - A review. Current Traditional Medicine, v. 1, n. 1, p. 5-17, jun. 2015.

HYACIENTH, Danilo Cabral; DE ALMEIDA, Sheylla Susan Moreira da Silva. Estudo fitoquímico, toxicidade em Artemia salina Leach e atividade antibacteriana de Pseudoxandra cuspidata Maas. Biota Amazônia, v. 5, n. 4, p. 4-7, out./dez. 2015.

NGUTA, Joseph Mwanzia et al. Biological screening of kenya medicinal plants using Artemia salina L. (Artemiidae). Pharmacologyonline, v. 2, n. 1, p. 458-78, ago. 2011. 
MEYER, Brian N. et al. Brine shrimp: a convenient general bioassay for active plant constituents. Journal of Medicinal Plants Research, v. 45, n. 5, p. 31-34, jan. 1982.

MOMIN, Mohammad Abdul Motalib et al. Phytopharmacological evaluation of ethanol extract of Sida cordifolia L. roots. Asian Pacific Journal of Tropical Biomedicine, v. 4, n. 1, p. 18-24, jan. 2014.

OLIVEIRA, Vitória Maria de et al. Uso de plantas medicinais por idosos. Anápolis Digital, v. 10, n. 1, p. 56-75, jan. 2020.

SILVA, Anna Erika Pinheiro et al. Avaliação tóxica, citotóxica, genotóxica e mutagênica Daturnera ulmifolia L. (chanana) em células eucarióticas. Revista Saúde em Foco, v. 2, n. 1, p. 25-48, jan./jul. 2015.

SILVA, Simoni Lobato da et al. Avaliação da toxicidade aguda pré-clínica do extrato metanólico das cascas do caule de Parahancornia amapa (Apocynaceae). Acta Amazonica, v. 46, n. 1, p. 73-80, jan./mar. 2016.

SIMÕES, Cláudia Maria Oliveira et al. (Orgs). Farmacognosia: da planta ao medicamento. 6.ed. Porto Alegre: Editora da UFRGS: Florianópolis: Editora da UFSC, 2010. 1104p.

SOBREIRA, Ana Laura Cabral et al. Investigação do potencial antifúngico de Sida planicaulis Cav. (Malvaceae) frente fungos filamentosos. Periódico Tchê-Química, v. 16, n. 31, p. 607-615, ago. 2019.

SOBREIRA, Ana Laura Cabral et al. Prospecção fitoquímica e avaliação antimicrobiana de Sida planicaulis Cav. (Malvaceae) sobre leveduras potencialmente patogênicas. Revista Verde de Agroecologia e Desenvolvimento Sustentável, v. 13, n. 3, p. 356-360, jul./set. 2018.

SOUZA, Júlio Cezar de et al. Produção de metabólitos secundários por meio da cultura de tecidos vegetais. Revista Fitos, v. 12, n. 3, p. 269-280, jan./jul. 2018. 